\title{
Rhabdomyolysis and exercise-associated hyponatremia in ultra-bikers and ultra-runners
}

\author{
Daniela Chlíbková ${ }^{1 \dagger}$, Beat Knechtle ${ }^{2^{*} \dagger}$, Thomas Rosemann², Ivana Tomášková ${ }^{3}$, Jan Novotný ${ }^{4}$, Alena Žákovská ${ }^{5}$ \\ and Tomáš Uher ${ }^{6}$
}

\begin{abstract}
Background: Exercise-associated hyponatremia $(E A H)$, rhabdomyolysis and renal failure appear to be a unique problem in ultra-endurance racers.

Methods: We investigated the combined occurrence of EAH and rhabdomyolysis in seven different ultra-endurance races and disciplines (i.e. multi-stage mountain biking, 24-h mountain biking, 24-h ultra-running and 100-km ultra-running).

Results: Two (15.4\%) ultra-runners (man and woman) from hyponatremic ultra-athletes ( $n=13)$ and four (4\%) ultra-runners (four men) from the normonatremic group $(n=100)$ showed rhabdomyolysis following elevated blood creatine kinase (CK) levels $>10,000 \mathrm{U} / \mathrm{L}$ without the development of renal failure and the necessity of a medical treatment. Post-race creatine kinase, plasma and urine creatinine significantly increased, while plasma $\left[\mathrm{Na}^{+}\right]$and creatine clearance decreased in hyponatremic and normonatremic athletes, respectively. The percentage increase of $\mathrm{CK}$ was higher in the hyponatremic compared to the normonatremic group $(P<0.05)$. Post-race CK levels were higher in ultra-runners compared to mountain bikers $(P<0.01)$, in faster normonatremic $(P<0.05)$ and older and more experienced hyponatremic ultra-athletes $(P<0.05)$. In all finishers, pre-race plasma $\left[K^{+}\right]$was related to post-race CK $(P<0.05)$.

Conclusions: Hyponatremic ultra-athletes tended to develop exercise-induced rhabdomyolysis more frequently than normonatremic ultra-athletes. Ultra-runners tended to develop rhabdomyolysis more frequently than mountain bikers. We found no association between post-race plasma $\left[\mathrm{Na}^{+}\right]$and $\mathrm{CK}$ concentration in both hypo- and normonatremic ultra-athletes.
\end{abstract}

Keywords: Mountain biking, Running, Long distances

\section{Introduction}

Exertional rhabdomyolysis, muscle cell breakdown with release of toxic chemicals into plasma and urine, is known as a relatively common response to prolonged strenuous exercise as running [1-21], but less frequently described for cycling [22-25] and triathlon [13]. Acute kidney injury is a potential and serious complication of severe exertional rhabdomyolysis $[5,6,11,18,20,21,24$, 26-28] and the prognosis is worse if renal failure develops [17-19, 26-29].

\footnotetext{
* Correspondence: beat.knechtle@hispeed.ch

${ }^{\dagger}$ Equal contributors

${ }^{2}$ Institute of Primary Care, University of Zurich, Zurich, Switzerland

Full list of author information is available at the end of the article
}

Factors proposed for the development of acute renal failure include dehydration secondary to inadequate fluid intake and/or diarrhoea or vomiting $[11,18,20,27]$, rhabdomyolysis [21, 27] and analgesic use [6, 11, 17, 20, 27, 29]. Aggressive drinking can lead to exercise-associated hyponatremia (EAH) and by a currently unknown mechanism induce muscle cell damage which is sufficient to cause kidney failure [6, 9, 14, 29]. According to Noakes [9], any level of overhydration will impair muscle cell function and will be sufficient to lead to a significant muscle cell damage. This can facilitate rhabdomyolysis and myoglobin release, leading to acute renal failure $[6,17,30]$. On the contrary, exertional muscle damage produced by eccentric exercise in

\section{() Biomed Central}

(c) 2015 Chlíbková et al. This is an Open Access article distributed under the terms of the Creative Commons Attribution License (http://creativecommons.org/licenses/by/4.0), which permits unrestricted use, distribution, and reproduction in any medium, provided the original work is properly credited. The Creative Commons Public Domain Dedication waiver (http:// creativecommons.org/publicdomain/zero/1.0/) applies to the data made available in this article, unless otherwise stated. 
healthy individuals can cause creatine kinase elevations without renal impairment $[1,2,7,8,31]$.

Previous studies related to a proposed link between rhabdomyolysis and EAH [3, 6-8, 14-17, 27, 32-36]. The simultaneous presentation of EAH and rhabdomyolysis creates a complicated and opposing treatment paradox with regard to fluid resuscitation $[6,8,27]$. There are two explanations for the development of EAH and rhabdomyolysis: one causes the other [14, 16, 27, 37 or they occur independently [14]. EAH could promote rhabdomyolysis through changes in intracellular $\left[\mathrm{K}^{+}\right]$and/or $\left[\mathrm{Ca}^{++}\right]$concentrations and reduce the stability of the cell membrane, or through direct mechanism decrease cell membrane stability through extracellular fluid osmotically drawn into the muscle cell $[6,14,27$, 35]. If the extracellular $\left[\mathrm{Na}^{+}\right]$falls during endurance exercise, the intracellular $\left[\mathrm{Ca}^{++}\right]$is impaired and increases of $\left[\mathrm{Ca}^{++}\right]$lead to cellular injury, because arginine vasopressin is not able to restore the $\left[\mathrm{Na}^{+}\right] /\left[\mathrm{Ca}^{++}\right]$balance [14]. During EAH, intracellular $\left[\mathrm{K}^{+}\right]$is depleted and transferred to the extracellular compartment and myocytes are injured [14]. EAH leads to cell swelling due to extracellular hypoosmolality and their reduction through extrusion of intracellular $\left[\mathrm{K}^{+}\right]$induces rhabdomyolysis [35]. On the contrary, rhabdomyolysis may cause EAH by arginine vasopressin secretion and fluid leakage into injured muscle [14, 27, 32-35]. Siegel [33] and also Kim et al. [12] confirmed the strong relationship between post-exercise interleukin- 6 and creatine kinase (CK) concentrations for support that rhabdomyolysis is a stimulus for EAH via the syndrome of inappropriate antidiuretic hormone secretion [33-35].

To prevent and treat rhabdomyolysis, it is necessary to measure CK levels as the most common marker to assess skeletal muscle damage $[7,12]$ which is also highly correlated to myoglobin $[7,38]$. The high CK concentrations could provide an explanation for the high incidence of EAH [36]. The peak CK concentration may be predictive of the development of renal failure [30]. The determination of other muscle enzymes aids less information for a reliable diagnosis [26].

Whether an association exists for the combination of EAH and rhabdomyolysis is currently under debate $[6,27]$. Therefore, in the present analysis, we aimed $(i)$ to identify athletes who developed simultaneously EAH and rhabdomyolysis. We aimed (ii) to expose possible biochemical factors in the hyponatremic and the normonatremic group to better explore this association.

\section{Methods}

\section{Participants}

Institutional review board approval was granted for this study by the local institutional ethics committee. The data were collected from 2012 to 2013 during individual ultra-running and ultra-cycling races. Participants were from the "Czech Championship 24-h mountain bike race" in Jihlava city from 2012 to 2013, the "Bike Race Marathon Rohozec 24 h" in Liberec city in 2012, the "Sri Chinmoy Self-Transcendence Marathon 24-h race" in Kladno city in 2012, the "Trilogy Mountain Bike Stage Race" in Teplice nad Metují in 2012 and 2013 and the 100-km running race in Plzen city in 2013, all held in the Czech Republic.

\section{Race details}

The "Czech Championship 24-h mountain bike race" took place during the second weekend in June 2012 and 2013 at 12:00 on Saturday and finished at 12:00 on Sunday. The course comprised a $9.5 \mathrm{~km}$ single-track with an elevation of $220 \mathrm{~m}$. The "Bike Race Marathon Rohozec $24 \mathrm{~h}$ " took place on June $9^{\text {th }}$ and finished on June $10^{\text {th }} 2012$. The course comprised a $12.6 \mathrm{~km}$ track with an elevation of $250 \mathrm{~m}$. In both races a single aid station located at the start/finish area was provided by the organizer with a variety of food and beverages. The ultra-mountain bikers were allowed to be supported by additional food and drinks in their pit stops. The "Sri Chinmoy Self-Transcendence Marathon 24-h race" took place from July $21^{\text {st }}$ to July $22^{\text {nd }} 2012$. The lap was $1 \mathrm{~km}$, situated around an athletic stadium on asphalt with $1 \mathrm{~m}$ change in altitude. The organizer provided the athletes with a buffet where both warm and cold food and beverages were offered. The "Trilogy Mountain Bike Stage Race" took place the first week in July 2012 and 2013. The prologue covered $3 \mathrm{~km}$ with $300 \mathrm{~m}$ difference in elevation. The first stage covered $66 \mathrm{~km}$ with $2,200 \mathrm{~m}$ of altitude to climb, the second stage was $63 \mathrm{~km}$ in length with $2,300 \mathrm{~m}$ difference in elevation and the third stage was $78.8 \mathrm{~km}$ with $3,593 \mathrm{~m}$. The "Czech championship 100-km running race" was held on July $9^{\text {th }} 2013$ and the ultra-runners had to run 66 times a lap of $1,500 \mathrm{~m}$ in length.

\section{Procedures, measurements and calculations}

At each race, blood and urine measurements were provided during registration the day before each race. Post-race measurements were upon completion of the race. Blood samples were drawn from the seated athlete into heparinized tubes via an antecubital vein. One Sarstedt S-Monovette (plasma gel, $7.5 \mathrm{ml}$ ) for chemical analysis was cooled, sent to the laboratory and analysed within $6 \mathrm{~h}$. Blood samples were obtained to determine pre- and post-race plasma $\left[\mathrm{Na}^{+}\right]$, plasma $\left[\mathrm{K}^{+}\right]$, plasma and urine creatinine and plasma CK. Plasma $\left[\mathrm{Na}^{+}\right]$, plasma $\left[\mathrm{K}^{+}\right]$, plasma creatinine and plasma $C K$ were determined using biochemical analyzer Modular SWA P300 (Roche, Basel, Switzerland). Samples of urine were collected in one Sarstedt Monovette for urine $(10 \mathrm{ml})$ 
and sent to the laboratory. CK was determined in urine samples using biochemical analyzer Modular SWA P300 (Roche, Basel, Switzerland).

\section{Statistical analysis}

Race performance was identical with the absolute ranking in each race due to the connection of runners, mountain bikers and various disciplines (i.e. 24-h race, stage race, $100-\mathrm{km}$ race). Continuous data underwent normality test with the D'Agostino and Pearson omnibus normality test. Because data did not pass normality testing, Spearman correlation analyses were used to examine the associations between blood and urinary parameters and pre-race characteristics within the hyponatremic group and the normonatremic group and all finishers. Paired sample $t$-tests or the Wilcoxon signed-rank tests (within different races and within genders, within all finishers, the normonatremic and the hyponatremic group) were used to compare parameters before and after the race as appropriate and to compare continuous measures. Mann-Whitney test was used for group comparisons (hyponatremic and normonatremic, ultra-runners and mountain bikers). Variables as post-race CK and type of race and post-race $\mathrm{CK}$ and gender were compared using one-way analysis of variance (ANOVA) and Tukey's multiple comparison tests. Statistical significance was set at $P<0.05$.

\section{Results}

Of the 145 ultra-runners and ultra-mountain bikers included in this study, 113 (81.8 \%) finishers underwent body mass measurements and provided pre and postrace blood and urine samples. Thirty-two ultra-athletes did not finish their race or they did not undergo all measurements. Thirteen (11.5 \%) finishers developed EAH, the remaining subjects $(n=100)$ were normonatremic. Two (1.8\%) ultra-runners (one man and one woman) from all finishers $(n=113)$, that means two $(15.4 \%)$ from the hyponatremic ultra-athletes $(n=13)$ and four (3.5\%) from all finishers and four (4\%) ultra-runners (four men) from the normonatremic group $(n=100)$ showed rhabdomyolysis following elevated blood CK levels $>10,000 \mathrm{U} / \mathrm{L}$ without the development of renal failure and the necessity of a medical treatment. Data of all cases with rhabdomyolysis (cases 1-6) are presented in Tables 1 and 2. Pre-race variables, age, race performance and body mass of present six cases with rhabdomyolysis including two ones with simultaneously EAH and rhabdomyolysis are shown in Table 1. Percentage changes in their blood and urine parameters (CK, plasma $\left[\mathrm{Na}^{+}\right]$, plasma $\left[\mathrm{K}^{+}\right]$, plasma creatinine, urine creatinine and creatinine clearance) are shown in Table 2.

We combined the racers by each ultra-endurance disciplines to four categories (SMTB - mountain bike stage racers, 24 MTB - 24-h ultra-mountain bikers, 24 RUN 24-h ultra-runners and 100RUN - 100-km ultra-runners) (Tables 3, 4, 5 and 6). The limitation was a small number of racers in some races or in genders due to the field nature of research and a toughness of such types of races. We included this fact into our conclusions and mentioned it in the discussion. Nevertheless, we divided racers also by genders to smaller groups to obtain a more accurate characterization of various endurance disciplines by post- minus pre-race differences in body mass, plasma $\left[\mathrm{Na}^{+}\right]$, plasma $\left[\mathrm{K}^{+}\right]$, plasma creatinine, urine creatinine and creatinine clearance (Tables 3, 4, 5 and 6). Post-race CK levels were significantly higher in ultrarunners $(n=31)$ compared to mountain bikers $(n=82)$ $(P<0.05)$. There was no difference in post-race plasma $\left[\mathrm{Na}^{+}\right]$between ultra-runners and mountain bikers $(P>0.05)$. The highest average post-race CK levels were in 24RUNners $(7,033 \pm 9,360 \mathrm{U} / \mathrm{L})$, follow by 100RUNners $(3,211 \pm 4,617 \mathrm{U} / \mathrm{L}), 24 \mathrm{MTBers}(1,123 \pm$ $1,077 \mathrm{U} / \mathrm{L})$ and SMTBers (918 $\pm 818 \mathrm{U} / \mathrm{L})$. In 24RUNners post-race increase of $\mathrm{CK}$ was significantly higher than in 24MTBers $(r=0.40, P<0.01)$.

We compared the hyponatremic $(n=13)$ and the normonatremic $(n=100)$ group of ultra-athletes. An average race performance (absolute order in each race) was $38.5 \pm$ 47.7 in the hyponatremic group and $49.5 \pm 43.3$ in the normonatremic group without significant difference between both groups. Race performance correlated negatively to post-race $(r=-0.37, P<0.05)$ and percentage change $(r=-0.31, P<0.05)$ in $C K$ only in the

Table 1 Age, body mass, training, pre-race experience, and race performance of subjects with rhabdomyolysis including case 1 and 2 (in bold) with EAH and rhabdomyolysis $(n=6)$

\begin{tabular}{|c|c|c|c|c|c|c|c|c|}
\hline Case & Age (yrs.) & Sex & $\begin{array}{l}\text { Change in } \\
\text { body mass (\%) }\end{array}$ & $\begin{array}{l}\text { Race } \\
\text { (discipline) }\end{array}$ & $\begin{array}{l}\text { Absolute } \\
\text { order }\end{array}$ & $\begin{array}{l}\text { Number of finished } \\
\text { ultra-marathons }(n)\end{array}$ & $\begin{array}{l}\text { Years as an active } \\
\text { biker runner) (yrs.) }\end{array}$ & $\begin{array}{l}\text { Training hours } \\
\text { weekly (h) }\end{array}$ \\
\hline 1 & 51 & $M$ & -2.9 & 100-km RUN & 19 & 4 & 27 & 12 \\
\hline 2 & 38 & $F$ & -2.6 & 24-km RUN & 1 & 30 & 13 & 15 \\
\hline 3 & 35 & M & -3.4 & 24-km RUN & 2 & 5 & 18 & 10 \\
\hline 4 & 31 & M & -0.9 & 24-km RUN & 20 & 7 & 5 & 15 \\
\hline 5 & 26 & M & -4.7 & 24-km RUN & 6 & 2 & 3 & 4 \\
\hline 6 & 48 & M & -0.2 & 100-km RUN & 2 & 3 & 5 & 11 \\
\hline
\end{tabular}


Table 2 Blood and urine parameters subjects with rhabdomyolysis including case 1 and 2 (in bold) with EAH with rhabdomyolysis $(n=6)$

\begin{tabular}{lllllllllll}
\hline Case & $\begin{array}{l}\text { PreR blood } \\
{\left[\mathrm{Na}^{+}\right](\mathrm{mmol} / \mathrm{L})}\end{array}$ & $\begin{array}{l}\text { PostR blood } \\
{\left[\mathrm{Na}^{+}\right](\mathrm{mmol} / \mathrm{L})}\end{array}$ & $\begin{array}{l}\text { Blood }\left[\mathrm{Na}^{+}\right] \\
\text {change }(\%)\end{array}$ & $\begin{array}{l}\text { Blood }\left[\mathrm{K}^{+}\right] \\
\text {change }(\%)\end{array}$ & $\begin{array}{l}\text { PreR blood } \\
\mathrm{CK} \mathrm{U/L}\end{array}$ & $\begin{array}{l}\text { PostR blood } \\
\mathrm{CK} \mathrm{U} / \mathrm{L}\end{array}$ & $\begin{array}{l}\text { Blood CK } \\
\text { change }(\%)\end{array}$ & $\begin{array}{l}\text { Blood } \mathrm{CR} \\
\text { change }(\%)\end{array}$ & $\begin{array}{l}\mathrm{CRCL} \\
\text { change }(\%)\end{array}$ & $\begin{array}{l}\text { Urine } C R \\
\text { change }(\%)\end{array}$ \\
\hline $\mathbf{1}$ & $\mathbf{1 3 8}$ & $\mathbf{1 3 4}$ & $\mathbf{- 2 . 9}$ & $\mathbf{2 3 . 5}$ & $\mathbf{5 0 9 . 4}$ & $\mathbf{1 4 5 1 2 . 9}$ & $\mathbf{2 7 4 9 . 0}$ & $\mathbf{2 5 . 0}$ & $\mathbf{- 2 2 . 4}$ & $\mathbf{1 0 2 . 5}$ \\
$\mathbf{2}$ & $\mathbf{1 3 7}$ & $\mathbf{1 3 3}$ & $\mathbf{- 2 . 9}$ & $\mathbf{- 2 9 . 2}$ & $\mathbf{1 3 0 . 0}$ & $\mathbf{1 5 1 7 2 . 9}$ & $\mathbf{1 1 5 7 1 . 5}$ & $\mathbf{1 4 . 3}$ & $\mathbf{- 1 4 . 7}$ & $\mathbf{1 6 5 . 6}$ \\
3 & 140 & 138 & -1.4 & -25.8 & 112.9 & 29702.9 & 26209.0 & 11.1 & -13.0 & 143.6 \\
4 & 138 & 138 & 0.0 & -40.7 & 150.6 & 12786.2 & 8378.2 & 10.0 & -9.9 & 212.7 \\
5 & 141 & 135 & -4.3 & -15.3 & 654.1 & 18085.9 & 2665.0 & 33.3 & -28.5 & 165.0 \\
6 & 137 & 141 & 2.9 & 36.7 & 321.9 & 20280.6 & 6381.5 & 50.0 & -33.4 & 485.3 \\
\hline
\end{tabular}

Note: PreR $=$ pre-race, Post $R=$ post-race, $\left[\mathrm{Na}^{+}\right]=$plasma sodium, $\left[\mathrm{K}^{+}\right]=$plasma potassium, $\mathrm{CK}=$ creatine kinase, $\mathrm{CR}=\mathrm{creatinine}, \mathrm{CRCL}=\mathrm{creatinine}$ clearance

normonatremic group. An average age of hyponatremic athletes was $39.5 \pm 6.9$ years, normonatremic athletes 38.6 \pm 8.5 years. Age was associated with post-race $(r=0.58$, $P<0.05)$ and percentage change $(r=0.61, P<0.05)$ in CK in the hyponatremic group. The number of years as an active runner (biker) was related to the percentage change $(r=0.75, P<0.05)$ in CK only in the hyponatremic group. Neither the number of finished ultra-marathons nor the total number of hours spent by training weekly, nor gender was related to post-race CK concentration $(P>0.05)$ in both groups. In the hyponatremic group, $\mathrm{CK}$ increased by $3,456 \pm 4,982 \mathrm{U} / \mathrm{L}$ and $1.9 \pm 2.9 \%$, respectively, from baseline $(P<0.01)$. In the normonatremic group CK increased by $1,831 \pm 4,073 \mathrm{U} / \mathrm{L}$ and $1.1 \pm 2.8 \%$, respectively, from baseline $(P<0.01)$. The percentage increase of CK was higher in the hyponatremic group $(P<0.05)$ (Fig. 1$)$.
Post-race $C K$ was related to pre-race plasma $\left[\mathrm{K}^{+}\right]$in all finishers $(P<0.05)$ (Fig. 2$)$. On the contrary, post-race or percentage change in CK was not associated with percentage change in body mass, post-race plasma $\left[\mathrm{Na}^{+}\right]$, plasma $\left[\mathrm{K}^{+}\right]$, plasma and urine creatinine and creatine clearance in all finishers, hyponatremic or normonatremic ultraathletes $(P>0.05)$.

In the hyponatremic group, post-race plasma $\left[\mathrm{Na}^{+}\right]$ decreased by $5.8 \pm 2.1 \mathrm{mmol} / \mathrm{L}(4.1 \pm 1.5 \%)(P<0.01)$. In the normonatremic group, post-race plasma $\left[\mathrm{Na}^{+}\right]$decreased by $1.2 \pm 2.4 \mathrm{mmol} / \mathrm{L},(0.8 \pm 1.7 \%) \quad(P<0.01)$. Percentage change in plasma $\left[\mathrm{Na}^{+}\right]$was significantly higher in hyponatremic ultra-athletes $(P<0.05)$. Plasma $\left[\mathrm{K}^{+}\right]$remained stable post-race $(P>0.05)$ in hyponatremic ultra-athletes. Post-race plasma $\left[\mathrm{K}^{+}\right]$decreased by $5.1 \pm 0.9 \mathrm{mmol} / \mathrm{L}$ post-race, $(1.3 \pm 25.3 \%)(P<0.05)$ in

Table 3 Age, anthropometric, blood and urine characteristics of the stage MTBers $(n=32)$

\begin{tabular}{|c|c|c|c|c|c|}
\hline & Pre-race & Post- race & Change in absolute & Change in percent & $P$-value \\
\hline \multicolumn{6}{|l|}{ Male multi-stage MTBers $(n=28)$} \\
\hline Age (yrs.) & $36.7 \pm 6.2$ & & & & \\
\hline Body mass (kg) & $76.6 \pm 6.6$ & $75.8 \pm 6.3$ & $-0.7 \pm 1.5^{*}$ & $-0.9 \pm 2.0^{*}$ & $<0.05$ \\
\hline Plasma sodium (mmol/L) & $142.8 \pm 1.9$ & $140.0 \pm 2.7$ & $-2.8 \pm 2.3^{* *}$ & $-1.9 \pm 1.6^{* *}$ & $<0.01$ \\
\hline Plasma potassium(mmol/L) & $4.4 \pm 0.7$ & $5.2 \pm 0.5$ & $0.8 \pm 0.9^{* *}$ & $21.3 \pm 21.7^{* *}$ & $<0.01$ \\
\hline Plasma creatinine (mg/dL) & $0.9 \pm 0.1$ & $1.2 \pm 0.5$ & $0.3 \pm 0.5^{*}$ & $33.3 \pm 64.8^{*}$ & $=0.01$ \\
\hline Plasma creatine kinase $(\mathrm{U} / \mathrm{L})$ & $180.7 \pm 95.9$ & $1009.4 \pm 812.6$ & $828.7 \pm 821.2^{* *}$ & $586.8 \pm 686.9^{* *}$ & $<0.01$ \\
\hline Plasma creatine clearance (ml/min) & $10849.4 \pm 1502.9$ & $8953.6 \pm 2447.9$ & $-1895.8 \pm 2158.7^{* *}$ & $-17.4 \pm 19.3^{* *}$ & $<0.01$ \\
\hline Urine creatinine (mmol/L) & $7.2 \pm 3.9$ & $19.7 \pm 17.6$ & $12.5 \pm 17.5^{* *}$ & $245.8 \pm 284.7^{* *}$ & $<0.01$ \\
\hline \multicolumn{6}{|l|}{ Female multi-stage MTBers $(n=4)$} \\
\hline Age (yrs.) & $39.0 \pm 7.2$ & & & & \\
\hline Body mass (kg) & $65.5 \pm 2.8$ & $63.2 \pm 2.8$ & $-2.3 \pm 1.1^{*}$ & $-3.5 \pm 1.6^{*}$ & $<0.05$ \\
\hline Plasma sodium (mmol/L) & $141.5 \pm 2.4$ & $138.8 \pm 1.7$ & $-2.8 \pm 2.2$ & $-1.9 \pm 1.6$ & 0.09 \\
\hline Plasma potassium(mmol/L) & $4.7 \pm 0.6$ & $4.8 \pm 0.4$ & $0.1 \pm 0.5$ & $1.8 \pm 10.6$ & 0.84 \\
\hline Plasma creatinine (mg/dL) & $0.8 \pm 0.1$ & $1.0 \pm 0.1$ & $0.2 \pm 0.1$ & $21.9 \pm 15.7$ & 0.68 \\
\hline Plasma creatine kinase $(\mathrm{U} / \mathrm{L})$ & $118.9 \pm 25.4$ & $418.1 \pm 413.3$ & $299.2 \pm 433.5$ & $308.1 \pm 454.3$ & 0.26 \\
\hline Plasma creatine clearance (ml/min) & $9862.8 \pm 712.2$ & $7943.8 \pm 1478.1$ & $-1918.9 \pm 1008.3^{*}$ & $-19.8 \pm 10.8^{*}$ & $<0.05$ \\
\hline Urine creatinine (mmol/L) & $7.2 \pm 2.6$ & $17.8 \pm 1.0$ & $10.6 \pm 2.6^{* *}$ & $191.7 \pm 159.6^{* *}$ & $<0.05$ \\
\hline
\end{tabular}

Note: Results are presented as mean $\pm \mathrm{SD} ;{ }^{*}=P<0.05,{ }^{* *}=P<0.01$ 
Table 4 Age, anthropometric, blood and urine characteristics of the 24-hr MTBers $(n=50)$

\begin{tabular}{|c|c|c|c|c|c|}
\hline & Pre-race & Post- race & Change in absolute & Change in percent & $P$-value \\
\hline \multicolumn{6}{|l|}{ Male 24-hr MTBers $(n=38)$} \\
\hline Age (yrs.) & $38.4 \pm 9.8$ & & & & \\
\hline Body mass (kg) & $78.8 \pm 7.7$ & $77.1 \pm 8.0$ & $-1.6 \pm 1.5^{* *}$ & $-2.0 \pm 2.1^{* *}$ & $<0.01$ \\
\hline Plasma sodium (mmol/L) & $138.0 \pm 1.6$ & $137.0 \pm 2.6$ & $-1.0 \pm 2.8^{*}$ & $-0.7 \pm 2.0^{*}$ & $<0.05$ \\
\hline Plasma potassium(mmol/L) & $5.5 \pm 1.0$ & $5.0 \pm 1.1$ & $-0.5 \pm 1.3^{*}$ & $-7.4 \pm 23.2^{*}$ & $<0.05$ \\
\hline Plasma creatinine (mg/dL) & $0.9 \pm 0.1$ & $1.2 \pm 0.2$ & $0.3 \pm 0.2^{* *}$ & $32.7 \pm 21.8^{* *}$ & $<0.01$ \\
\hline Plasma creatine kinase (U/L) & $207.5 \pm 92.3$ & $1316.3 \pm 1111.9$ & $1108.9 \pm 1099.1^{* *}$ & $590.9 \pm 560.4^{* *}$ & $<0.01$ \\
\hline Plasma creatine clearance (ml/min) & $10657.2 \pm 1614.2$ & $9623.2 \pm 1837.4$ & $-1034.1 \pm 1121.0^{*}$ & $-9.8 \pm 11.7^{*}$ & $<0.05$ \\
\hline Urine creatinine (mmol/L) & $7.9 \pm 3.7$ & $18.4 \pm 5.7$ & $10.5 \pm 6.5^{* *}$ & $212.3 \pm 236.4^{* *}$ & $<0.01$ \\
\hline \multicolumn{6}{|l|}{ Female 24-hr MTBers $(n=12)$} \\
\hline Age (yrs.) & $37.7 \pm 7.4$ & & & & \\
\hline Body mass (kg) & $62.1 \pm 5.1$ & $60.0 \pm 5.4$ & $-1.9 \pm 3.1^{*}$ & $-2.7 \pm 5.1^{*}$ & $<0.05$ \\
\hline Plasma sodium (mmol/L) & $137.5 \pm 2.5$ & $136.6 \pm 2.0$ & $-0.9 \pm 3.0$ & $-0.6 \pm 2.2$ & 0.31 \\
\hline Plasma potassium(mmol/L) & $5.1 \pm 0.8$ & $4.7 \pm 0.6$ & $-0.5 \pm 0.7^{*}$ & $-8.2 \pm 12.0^{*}$ & $<0.05$ \\
\hline Plasma creatinine (mg/dL) & $0.9 \pm 0.1$ & $1.0 \pm 0.1$ & $0.1 \pm 0.2$ & $13.4 \pm 20.0$ & 0.05 \\
\hline Plasma creatine kinase (U/L) & $148.7 \pm 75.7$ & $568.9 \pm 293.4$ & $420.2 \pm 285.3^{* *}$ & $349.9 \pm 343.7^{* *}$ & $<0.01$ \\
\hline Plasma creatine clearance (ml/min) & $9365.7 \pm 1579.9$ & $8228.8 \pm 2228.7$ & $-13.0 \pm 17.3$ & $-12.5 \pm 15.7$ & 0.05 \\
\hline Urine creatinine (mmol/L) & $5.4 \pm 2.4$ & $15.7 \pm 4.9$ & $10.3 \pm 4.4^{* *}$ & $252.4 \pm 207.8^{* *}$ & $<0.01$ \\
\hline
\end{tabular}

Note: Results are presented as mean $\pm \mathrm{SD} ;{ }^{*}=P<0.05,{ }^{* *}=P<0.01$

Table 5 Age, anthropometric, blood and urine characteristics of the 24-hr ultra-runners $(n=12)$

\begin{tabular}{|c|c|c|c|c|c|}
\hline & Pre-race & Post- race & Change in absolute & Change in percent & $P$-value \\
\hline \multicolumn{6}{|l|}{ Male 24-hr ultra-runners $(n=8)$} \\
\hline Age (yrs.) & $37.5 \pm 8.1$ & & & & \\
\hline Body mass (kg) & $71.1 \pm 5.9$ & $70.0 \pm 6.7$ & $-1.1 \pm 1.2^{*}$ & $-1.6 \pm 1.8^{*}$ & $<0.05$ \\
\hline Plasma sodium (mmol/L) & $140.1 \pm 1.2$ & $139.1 \pm 2.0$ & $-1.0 \pm 2.3$ & $-0.7 \pm 1.7$ & 0.26 \\
\hline Plasma potassium(mmol/L) & $6.7 \pm 1.1$ & $5.1 \pm 0.6$ & $-1.6 \pm 1.3^{*}$ & $-21.8 \pm 17.4^{*}$ & $<0.05$ \\
\hline Plasma creatinine (mg/dL) & $0.9 \pm 0.1$ & $0.9 \pm 0.2$ & $0.0 \pm 0.1$ & $10.6 \pm 12.7$ & 0.06 \\
\hline Plasma creatine kinase $(\mathrm{U} / \mathrm{L})$ & $224.0 \pm 192.0$ & $8813.8 \pm 10536.4$ & $8589.8 \pm 10500.7^{*}$ & $5782.1 \pm 8659.2^{*}$ & $<0.05$ \\
\hline Plasma creatine clearance (ml/min) & $10657.2 \pm 1614.2$ & $9623.2 \pm 1837.4$ & $-1034.1 \pm 1121.0^{*}$ & $-9.8 \pm 11.7^{*}$ & $<0.05$ \\
\hline Urine creatinine $(\mathrm{mmol} / \mathrm{L})$ & $7.5 \pm 1.3$ & $17.5 \pm 2.6$ & $10.0 \pm 2.7^{* *}$ & $139.2 \pm 47.9^{* *}$ & $<0.01$ \\
\hline \multicolumn{6}{|l|}{ Female 24-hr ultra-runners $(n=4)$} \\
\hline Age (yrs.) & $40.0 \pm 7.7$ & & & & \\
\hline Body mass (kg) & $56.9 \pm 5.2$ & $56.3 \pm 5.1$ & $-0.6 \pm 0.6$ & $-1.0 \pm 1.2$ & 0.16 \\
\hline Plasma sodium (mmol/L) & $139.8 \pm 2.6$ & $137.8 \pm 3.4$ & $-2.0 \pm 3.4$ & $-1.4 \pm 2.4$ & 0.32 \\
\hline Plasma potassium(mmol/L) & $6.2 \pm 0.3$ & $4.6 \pm 0.2$ & $-1.7 \pm 0.2^{* *}$ & $-26.4 \pm 2.9^{* *}$ & $<0.01$ \\
\hline Plasma creatinine (mg/dL) & $0.7 \pm 0.1$ & $0.7 \pm 0.1$ & $0.01 \pm 0.0$ & $9.0 \pm 7.3$ & 0.18 \\
\hline Plasma creatine kinase $(\mathrm{U} / \mathrm{L})$ & $99.0 \pm 23.5$ & $5507.1 \pm 6600.4$ & $5408.1 \pm 6580.2^{* *}$ & $4765.0 \pm 4806.5^{* *}$ & 0.19 \\
\hline Plasma creatine clearance (ml/min) & $10353.4 \pm 795.3$ & $9547.0 \pm 845.9$ & $-806.4 \pm 838.7$ & $-7.6 \pm 7.9$ & 0.15 \\
\hline Urine creatinine $(\mathrm{mmol} / \mathrm{L})$ & $4.5 \pm 2.1$ & $15.2 \pm 0.2$ & $10.7 \pm 1.9^{* *}$ & $327.8 \pm 276.6^{* *}$ & $<0.01$ \\
\hline
\end{tabular}


Table 6 Age, anthropometric, blood and urine characteristics of the 100-km ultra-runners ( $n=19)$

\begin{tabular}{|c|c|c|c|c|c|}
\hline & Pre-race & Post- race & Change in absolute & Change in percent & $P$-value \\
\hline \multicolumn{6}{|l|}{ Male $100 \mathrm{~km}$ ultra-runners $(n=4)$} \\
\hline Age (yrs.) & $43.9 \pm 9.6$ & & & & \\
\hline Body mass (kg) & $67.0 \pm 8.5$ & $65.1 \pm 7.8$ & $-1.8 \pm 1.3^{* *}$ & $-2.6 \pm 1.7^{* *}$ & $<0.01$ \\
\hline Plasma sodium (mmol/L) & $139.6 \pm 2.2$ & $137.8 \pm 2.9$ & $-1.8 \pm 3.6$ & $-1.3 \pm 2.6$ & 0.09 \\
\hline Plasma potassium(mmol/L) & $6.2 \pm 1.0$ & $5.6 \pm 0.7$ & $-0.6 \pm 1.6$ & $-6.2 \pm 24.9$ & 0.18 \\
\hline Plasma creatinine (mg/dL) & $0.9 \pm 0.2$ & $1.1 \pm 0.3$ & $0.2 \pm 0.3$ & $25.8 \pm 48.1$ & 0.05 \\
\hline Plasma creatine kinase $(\mathrm{U} / \mathrm{L})$ & $227.1 \pm 148.9$ & $4357.1 \pm 5822.5$ & $4130.0 \pm 5742.7^{*}$ & $1925.5 \pm 2180.3^{*}$ & $<0.05$ \\
\hline Plasma creatine clearance (ml/min) & $8985.3 \pm 2219.0$ & $7327.7 \pm 2080.2$ & $-1657.5 \pm 2146.8^{* *}$ & $-16.7 \pm 17.9^{*}$ & $<0.05$ \\
\hline Urine creatinine $(\mathrm{mmol} / \mathrm{L})$ & $6.6 \pm 4.6$ & $18.5 \pm 4.1$ & $11.9 \pm 5.8^{* *}$ & $365.2 \pm 332.1^{* *}$ & $<0.01$ \\
\hline \multicolumn{6}{|l|}{ Female 100 km ultra-runners $(n=5)$} \\
\hline Age (yrs.) & $40.6 \pm 5.8$ & & & & \\
\hline Body mass (kg) & $59.0 \pm 7.7$ & $57.6 \pm 6.6$ & $-1.4 \pm 1.2$ & $-2.3 \pm 1.6$ & 0.05 \\
\hline Plasma sodium (mmol/L) & $138.4 \pm 2.3$ & $136.0 \pm 1: 6$ & $-2.4 \pm 2.7$ & $-1.7 \pm 1.9$ & 0.12 \\
\hline Plasma potassium(mmol/L) & $5.2 \pm 0.9$ & $5.5 \pm 0.9$ & $0.3 \pm 0.6$ & $7.3 \pm 13.0$ & 0.28 \\
\hline Plasma creatinine (mg/dL) & $0.8 \pm 0.1$ & $1.0 \pm 0.2$ & $0.2 \pm 0.1^{*}$ & $25.6 \pm 20.6^{*}$ & $<0.05$ \\
\hline Plasma creatine kinase $(\mathrm{U} / \mathrm{L})$ & $251.1 \pm 187.7$ & $1949.3 \pm 1357.3$ & $1698.2 \pm 1413.2^{*}$ & $990.7 \pm 748.9^{*}$ & 0.06 \\
\hline Plasma creatine clearance (ml/min) & $9335.1 \pm 1748.4$ & $7463.4 \pm 2240.3$ & $-1871.7 \pm 1199.4^{*}$ & $-20.6 \pm 12.9^{*}$ & $<0.05$ \\
\hline Urine creatinine $(\mathrm{mmol} / \mathrm{L})$ & $8.2 \pm 4.8$ & $19.4 \pm 7.4$ & $11.2 \pm 6.7^{*}$ & $192.2 \pm 153.0^{*}$ & $<0.05$ \\
\hline
\end{tabular}

Note: Results are presented as mean $\pm \mathrm{SD} ;{ }^{*}=P<0.05,{ }^{* *}=P<0.01$

normonatremic ultra-athletes. Pre-race plasma $\left[\mathrm{K}^{+}\right]$was not related to post-race plasma $\left[\mathrm{K}^{+}\right]$in all finishers, hyponatremic or normonatremic ultra-athletes $(P>0.05)$. Post-race plasma creatinine increased by $1.1 \pm 0.5 \mathrm{mg} / \mathrm{dL}$ $(39.5 \pm 71.0 \%) \quad(P<0.05)$ in hyponatremic finishers. Post-race plasma creatinine increased by $1.1 \pm 0.3 \mathrm{mg} /$ $\mathrm{dL}(25.2 \pm 34.1 \%)(P<0.01)$ in normonatremic finishers. Post-race urine creatinine increased by $7.9 \pm 7.0 \mathrm{mmol} / \mathrm{L}$ $(216 \pm 287 \%)(P<0.01)$ in the hyponatremic group. Post-race urine creatinine increased by $9.5 \pm 5.7 \mathrm{mmol} / \mathrm{L}$
$(229 \pm 235 \%)(p<0.01)$ in the normonatremic group. Post-race plasma creatine clearance decreased by 2,454 $\pm 2,428 \mathrm{ml} / \mathrm{min}(21.7 \pm 18.4 \%)(P<0.01)$ in the hyponatremic group. Plasma creatine clearance decreased by $1,913 \pm 1,815 \mathrm{ml} / \mathrm{min}(18.0 \pm 15.6 \%)(P<0.01)$ in the normonatremic group. In comparison of the normonatremic and the hyponatremic group percentage change in plasma $\left[\mathrm{K}^{+}\right]$, plasma and urine creatinine and creatine clearance did not differ significantly between both groups.
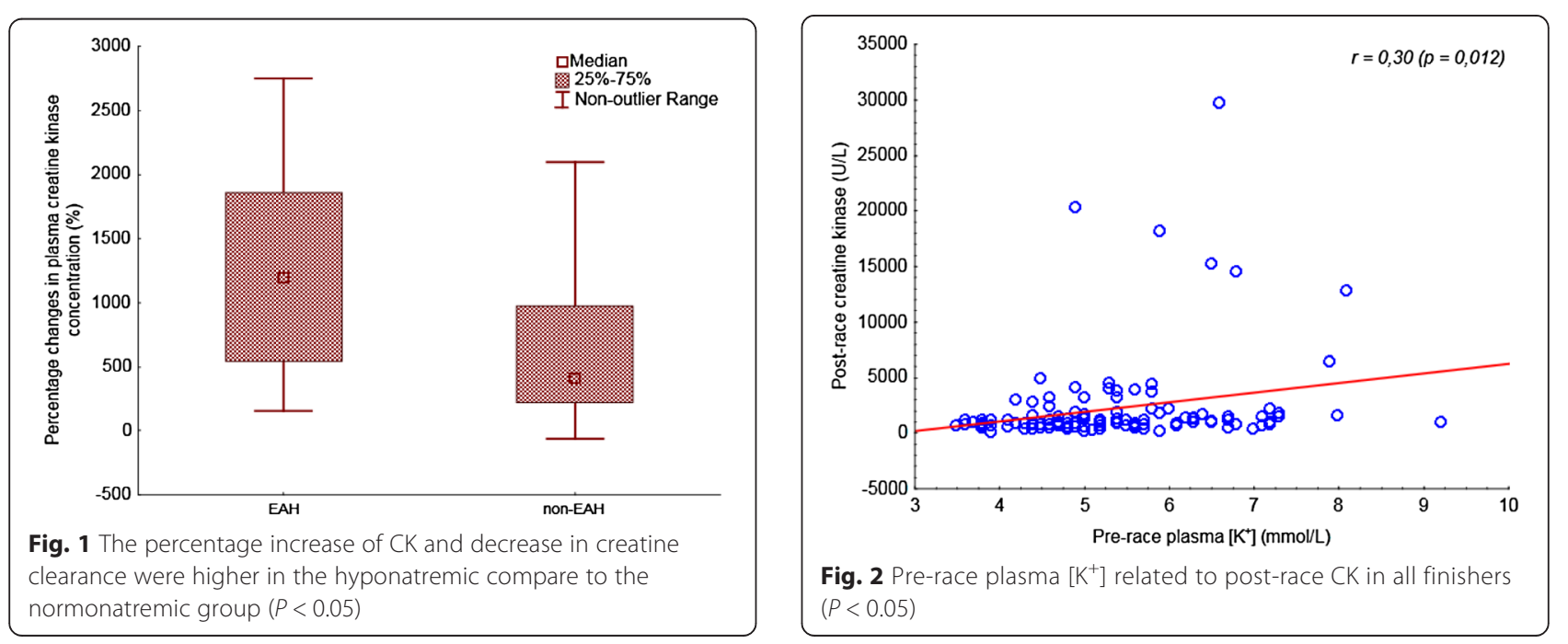


\section{Discussion}

The most important result was that two (15.4\%) ultrarunners from 13 hyponatremic and four $(4.0 \%)$ ultrarunners from 100 normonatremic finishers $(5.3 \%$ from a total number of finishers) developed post-race CK levels higher than 10,000 U/L without the occurrence of renal failure and the necessity of a medical treatment.

\section{Pre-race characteristics of cases with rhabdomyolysis (cases 1-6)}

The present case 1 (51-year-old man) and case 2 (38-yearold woman) with EAH and rhabdomyolysis were among the faster, but not younger finishers in their races. Female 24RUNner case 2 was even the first according to the absolute order among both genders. The $153-\mathrm{km}$ ultra-runners with EAH and rhabdomyolysis (three men and one woman) exhibited an average age of $38 \pm 8$ years in a study by Ellis et al. [14]. In a recent study published by Hoffman et al. [8], a 161-km hyponatremic ultra-runner with rhabdomyolysis was 53 years old. In the study of Boulter et al. [17], three (from four with acute renal failure) hyponatremic male $89-\mathrm{km}$ ultra-marathoners noted their average age of $34.0 \pm 8.7$ years. In the study of Bruso et al. [6], 161-km ultra-runners with EAH and rhabdomyolysis were men with an average age of $39 \pm 7$ years and they tended to be younger and faster than those not developing $\mathrm{EAH}$ with rhabdomyolysis. The present normonatremic male cases with rhabdomyolysis (cases 3-6) with mean age $35.0 \pm 9.4$ were among the faster finishers and they were on average younger compared to the rest of the normonatremic athletes. As described by Hoffman et al. [7] in their study of 161-km finishers of "Western States Endurance Run" (WSER) with rhabdomyolysis, blood CK concentrations were not related to finish time, age or the number of prior similar completed races. The comparison of the present hyponatremic cases $(1,2)$ and normonatremic cases (3-6) with rhabdomyolysis was impossible due to the low number of cases. Nevertheless, when we compared all hyponatremic and normonatremic cases, the present older and more trained (i.e. more years spent by running/biking) hyponatremic ultra-athletes developed higher post-race CK concentrations than the younger ones and the less trained hyponatremic ultra-athletes. Moreover, the present faster normonatremic finishers developed higher post-race CK concentrations than the slower normonatremic finishers. On the contrary, race experience (i.e. the number of finished ultra-marathons) or the training frequency and length (i.e. number of training hours per week) related to an increased CK neither in hyponatremic, nor in normonatremic ultra-athletes. Gender was not related to $\mathrm{CK}$ in the present ultra-athletes. The rare incidence of women with EAH and rhabdomyolysis probably reflects the ratio of female to male finishers in similar ultra-endurance races [6].

\section{Creatine kinase concentrations}

Normal CK post-race values are up to $~ 2,000$ U/L [9]. In accordance to Sinert et al. [31], inclusion criteria were an elevated CK of more than $500 \mathrm{U} / \mathrm{L}$ and they reported exertional rhabdomyolysis with admission CK levels between $700 \mathrm{U} / \mathrm{L}$ and $167,000 \mathrm{U} / \mathrm{L}$. CK levels of roughly $500-1500 \mathrm{U} / \mathrm{L}$ [24] or approximately over $2,000 \mathrm{U} / \mathrm{L}$ are used as the criterion for statin myopathy $[39,40]$ and CK above 10,000 $\mathrm{U} / \mathrm{L}$ as diagnosis of rhabdomyolysis [27, 31]. In the present study, $\mathrm{CK} \geq 2,000 \mathrm{U} / \mathrm{L}$ and associated myopathy developed three (23.1 \%) hyponatremic (one male and one female 100RUNners and one male 24MTBer) and fifteen (15\%) normonatremic (six male 24MTBers, two male and one female 24RUNners, three male and one female 100RUNners and two male SMTBers) ultraathletes. Overall, eighteen (15.9 \%) of all present ultraathletes $(n=113)$ developed exercise-associated myopathy.

Cases 1 and 2 developed biochemical EAH and exerciseinduced rhabdomyolysis with CK levels of 14,512 U/L and $15,172 \mathrm{U} / \mathrm{L}$, respectively. The rest of the hyponatremic group $(n=11)$ had CK range from $691 \mathrm{U} / \mathrm{L}$ to $3,163 \mathrm{U} / \mathrm{L}$. In the normonatremic cases 3, 5 and 6 with rhabdomyolysis post-race $\mathrm{CK}$ increased in the range from $12,768 \mathrm{U} / \mathrm{L}$ to 20,280 U/L. Aside from cases 1 and 2, normonatremic cases 3, 5 and 6 exhibited CK concentrations higher than $14,512 \mathrm{U} / \mathrm{L}$, the lower initial post-race value of two cases with EAH and rhabdomyolysis. The study of the $153-\mathrm{km}$ ultra-runners with EAH and rhabdomyolysis described CK levels in the range from 15,636 U/L to more than 100,000 U/L [14]. Rhabdomyolysis in combination with EAH in one male athlete was presented by Putterman [35] with CK 1,545 U/L which peaked at 10,300 U/L. Boulter et al. [17] described in three $89-\mathrm{km}$ runners with EAH and rhabdomyolysis CK levels in a wide range from 5,718 U/L to 48,934 U/L. Hoffman et al. [16] observed higher blood CK concentrations among those with EAH than those not developing EAH at the 2011 WSER. In 161-km ultra-run race mean CK concentrations were even 54,583 U/L in the hyponatremic group and 30,335 $\mathrm{U} / \mathrm{L}$ in the normonatremic group [16]. Percentage change in CK was significantly higher in the present hyponatremic compare to normonatremic ultra-athletes. Hyponatremic finishers with an average post-race CK 3,658 $\pm 5,029 \mathrm{U} / \mathrm{L}$ tended to develop exercise induced rhabdomyolysis more than present normonatremic ultra-athletes with an average post-race CK $2,025 \pm 4,094 \mathrm{U} / \mathrm{L}$.

We could not compare validly different kinds of races and ultra-disciplines due to the small number of participants in some observed races. Nevertheless, post-race plasma CK significantly increased in male and female finishers in all races (SMTB, 24MTB, 24RUN and 100RUN), except female SMTBers with a non-significant increase. Notwithstanding, in comparison of all ultra-runners $(n=31)$ and all mountain bikers $(n=82)$ post-race CK 
levels were significantly higher in the present ultrarunners. Despite a dissimilar number of participants in each race discipline we found the highest post-race CK levels in the 24RUNners and the 100RUNners with a significantly higher increase of post-race CK in 24RUNners compared to 24MTBers. Moreover, cases 1 and 6 were from 100RUN and cases 2, 3, 4 and 5 were 24RUNners. In accordance to Hoffman et al. [8] mild to moderate elevations of CK are common in long running distance and exertional rhabdomyolysis is often associated with EAH. Skenderi et al. [1] assumed that prolonged exercise at even moderate intensity can induce asymptomatic exertional rhabdomyolysis. In their study of $246-\mathrm{km}$ ultra-runners an increase of post-race CK was 43,763 U/L; nevertheless, the ultra-runners did not require hospitalisation. The reasons for an increase in CK could be also the duration of races and the large eccentric component of ultra-running races $[7,12]$. On the contrary, a low increase of CK of $542 \mathrm{U} / \mathrm{L}$ during $24 \mathrm{~h}$ after the exercise appeared after two hours of cycling [25]. The groups of ultra-runners and MTBers were not equal; nevertheless, the present ultra-runners tended to develop more frequently exercise-induced rhabdomyolysis than the present ultra-MTBers.

Normonatremic cases 3 and $6(1.8 \%$ from the total of 113 ultra-athletes) developed post-race CK concentration of 20,280 U/L, a level associated with renal failure [31]. Acute kidney injury is a complication of severe rhabdomyolysis ( $\mathrm{CK}>60,000 \mathrm{U} / \mathrm{L}$ to $80,000 \mathrm{U} / \mathrm{L}$ ) and the prognosis is worse with renal failure [38]. However, no present finisher developed acute kidney injury or renal failure or need a medical treatment. In the present study the post-race CK levels were not as high as in other studies. However, the average increase was from $2,665 \%$ to $26,209 \%$ in all cases with rhabdomyolysis. Factors associated with acute renal failure include rhabdomyolysis with CK concentrations higher than $20,000 \mathrm{U} / \mathrm{L}$ or higher than five times the normal value [31, 38]. However, no defined level exists. Following Meijer et al. [30], the risk of acute renal injury in rhabdomyolysis is low at CK levels lower than 15,000 U/L to $20,000 \mathrm{U} / \mathrm{L}$. Acute renal injury with CK levels at 5,000 U/L usually occurs with hypovolemia (low circulating volume) or aciduria (acidic urine) [26, 27, 29, 31]. However, exercise may induces factors protect against hypovolemia and aciduria. Exercise-induced rhabdomyolysis with mean CK concentrations up to $40,000 \mathrm{U} / \mathrm{L}[1,31]$ has not been reliable to diagnose renal failure [17]. Blood CK concentration was reported from the 161-km WSER in 1980 through 1983 [3, 4], 1995 [5], 2009 [6] and 2010 [7] and Bruso et al. [6] first defined the relationship between EAH and rhabdomyolysis in the $161-\mathrm{km}$ ultrarun in five runners with CK values of 40,000 U/L. CK concentrations in the 2010 WSER finishers were higher than values previously reported [7]. Hoffman et al. [7] suggested that stress caused by trail running race with its challenging conditions produced severe muscle damage. However, CK values higher than 20,000 U/L are common for this type of event and seldom result in detrimental consequences. Hoffman et al. [7] found no athlete with acute renal failure, despite high mean CK levels of 32,956 U/L. Thirty-nine ultra-runners finishing a $245-\mathrm{km}$ race with $\mathrm{CK}$ values exceeding 40,000 U/L also had not been shown to have acute renal failure [1]. On the contrary, four cases of acute renal failure in the Comrades marathon had various levels of $\mathrm{CK}$ values of 39,000 U/L, 29, $800 \mathrm{U} / \mathrm{L}, 24,120 \mathrm{U} / \mathrm{L}$ and 2,220 U/L [17]. In the cohort of twenty-six patients with severe rhabdomyolysis the average level of CK with $38,351 \mathrm{U} / \mathrm{L}$ was predicted the development of acute renal failure [30]. Nevertheless, exercise-induced rhabdomyolysis rarely progresses to acute renal failure [27, 31] and less severe forms of rhabdomyolysis or in cases of hyperCKemia (i.e. chronic or intermittent muscle destruction) present with no renal failure [26]. Moreover, exertional muscle damage produced by eccentric exercise can lead to an elevated CK without renal impairment $[2,39]$. CK level is, therefore, not useful in distinguishing acute renal failure [2, 31, 41]. Given the wide range of CK levels the value of CK is limited to diagnose rhabdomyolysis [30]. Factors for renal failure in cases of exertional rhabdomyolysis in marathon running could be a pre-existing viral/bacterial infection, heat stress, dehydration, latent myopathy, NSAID (non-steroidal antiinflammatory drugs), other drugs or analgesic use [2, $27,31]$. In the present study we were not able to observe these factors in ultra-athletes. However, the present results support hypothesis that the magnitude of elevated CK do not have exactly and always predict acute renal failure $[2,31,38,39,41]$.

\section{Plasma $\left[\mathrm{Na}^{+}\right]$and $\left[\mathrm{K}^{+}\right]$concentrations}

Plasma $\left[\mathrm{Na}^{+}\right]$decreased in cases 1 and 2 with rhabdomyolysis and EAH and cases 3 and 5 with rhabdomyolysis within the hyponatremic and the normonatremic group with a significantly higher increase in the hyponatremic group. In different kinds of the present races limited by various numbers of participant's plasma $\left[\mathrm{Na}^{+}\right]$decreased in all ultra-disciplines. Hyponatremia as the most common electrolyte disorder associated with ultra-running and muscle-cell swelling with mechanical stress caused by running (footrace) may result in skeletal muscle damage, rhabdomyolysis, or acute renal injury [27]. However, we found no study about the occurrence of rhabdomyolysis and EAH in cycling races. Also the present post-race CK levels were significantly higher in the ultra-runners and no mountain biker presented EAH with rhabdomyolysis or just rhabdomyolysis. Blood $\left[\mathrm{Na}^{+}\right]$and CK concentrations 
were negatively correlated in $161-\mathrm{km}$ ultra-runners [16]. In all present finishers $(n=113)$, in hyponatremic and in normonatremic finishers, $\mathrm{CK}$ concentrations were not associated with plasma $\left[\mathrm{Na}^{+}\right]$. In a recent study of Hoffman and Stuempfle [15] from the WSER in 2011 and 2013, a significant relationship between plasma $\left[\mathrm{Na}^{+}\right]$and $\mathrm{CK}$ concentration was also evident, however, without the difference between the hyponatremic and the normonatremic group due to wide variability in creatine kinase concentrations. Similarly, in the study of Hoffman et al. [7], the relationship between blood $\mathrm{CK}$ and $\left[\mathrm{Na}^{+}\right]$did not reach statistical significance.

The recognition of electrolyte abnormalities associated with rhabdomyolysis and induced acute kidney injury like hyperkalemia (serum $\left[\mathrm{K}^{+}\right] \geq 5.5 \mathrm{mmol} / \mathrm{L}$ ) is important to remove $\left[\mathrm{K}^{+}\right]$from the body [26]. Hyperkalemia can be classified according to serum $\left[\mathrm{K}^{+}\right]$into mild (5.5 - $6.5 \mathrm{mmol} / \mathrm{L})$, moderate $(6.5-7.5 \mathrm{mmol} / \mathrm{L})$ and severe (>7.5 mmol/L) hyperkalemia [42]. A metabolic disorder known to cause EAH and rhabdomyolysis is also hypokalemia, when $\left[\mathrm{K}^{+}\right]$depletion due to cell swelling eventually induces rhabdomyolysis [35]. None of present ultra-athletes showed post-race hypokalemia. Present case 1 developed post-race plasma $\left[\mathrm{K}^{+}\right] 5.2 \mathrm{mmol} / \mathrm{L}$ and case 2 plasma $\left[\mathrm{K}^{+}\right]$of $4.6 \mathrm{mmol} / \mathrm{L}$. A study of four athletes with EAH and rhabdomyolysis showed plasma $\left[\mathrm{K}^{+}\right]$ in the range from $4.1 \mathrm{mmol} / \mathrm{L}$ to $4.9 \mathrm{mmol} / \mathrm{L} \mathrm{[14]}$. The present levels of post-race plasma $\left[\mathrm{K}^{+}\right]$were higher than in the study of Bruso et al. [6] with an average of $4.0 \mathrm{mmol} / \mathrm{L}$ (range $3.4-4.9 \mathrm{mmol} / \mathrm{L}$ ) in his 5 cases with EAH and rhabdomyolysis and higher than in the study of Boulter et al. [17] with an average of $4.4 \mathrm{mmol} / \mathrm{L}$.

The interesting finding was that the present cases 1 and 2 showed pre-race values of plasma $\left[\mathrm{K}^{+}\right](6.8 \mathrm{mmol} / \mathrm{L}$ and $6.5 \mathrm{mmol} / \mathrm{L}$, respectively) which tended to be higher than in other hyponatremic ultra-athletes. In the present cases with just rhabdomyolysis (cases $3-5$ ), post-race plasma [K ${ }^{+}$] ranged from $4.8 \mathrm{mmol} / \mathrm{L}$ to $5.0 \mathrm{mmol} / \mathrm{L}$, only case 6 reached $6.7 \mathrm{mmol} / \mathrm{L}$ (moderate post-race hyperkalemia). Another finding was the post-race decrease of plasma $\left[\mathrm{K}^{+}\right]$ in all cases (1-5), except case 6 . The reason could be probably pre-race mild to severe hyperkalemia in cases $1-5$ due to the range of pre-race plasma $\left[\mathrm{K}^{+}\right]$from $5.9 \mathrm{mmol} / \mathrm{L}$ to $8.1 \mathrm{mmol} / \mathrm{L}$ (case 4 with $8.1 \mathrm{mmol} / \mathrm{L}$ ). On the contrary, case 6 presented with a pre-race level of $4.9 \mathrm{mmol} / \mathrm{L}$ and therefore probably exhibited postrace increase of plasma $\left[\mathrm{K}^{+}\right]$. The average post-race plasma $\left[\mathrm{K}^{+}\right]$in the present normonatremic finishers was in the range from $3.8 \mathrm{mmol} / \mathrm{L}$ to $8.2 \mathrm{mmol} / \mathrm{L}$ and twenty-seven $(27 \%)$ athletes developed levels higher than $5.5 \mathrm{mmol} / \mathrm{L}$. Their average pre-race plasma $\left[\mathrm{K}^{+}\right]$ was $5.4 \pm 1.2 \mathrm{mmol} / \mathrm{L}$ (range $3.5 \mathrm{mmol} / \mathrm{L}$ to $9.2 \mathrm{mmol} / \mathrm{L}$ ) with fifteen $(15 \%)$ ultra-athletes with pre-race level $\geq$ $5.5 \mathrm{mmol} / \mathrm{L}$. Hyponatremic finishers had an average post-race plasma $\left[\mathrm{K}^{+}\right]$in the range from $4.4 \mathrm{mmol} / \mathrm{L}$ to $6.5 \mathrm{mmol} / \mathrm{L}$ with four $(30.8 \%)$ finishers with a level higher than $5.5 \mathrm{mmol} / \mathrm{L}$. Even in five $(38.5 \%)$ hyponatremic finishers pre-race plasma $\left[\mathrm{K}^{+}\right]$reached levels of $\geq 5.5 \mathrm{mmol} / \mathrm{L}$. Post-race plasma $\left[\mathrm{K}^{+}\right]$significantly decreased in both genders in 24MTBers and 24RUNners and non-significantly in male 100RUNners despite the limitation of different number of finishers in each ultraendurance discipline.

Another finding was that pre-race plasma $\left[\mathrm{K}^{+}\right]$showed an average value of $6.7 \mathrm{mmol} / \mathrm{L}$ and $6.2 \mathrm{mmol} / \mathrm{L}$ in male and female 24RUNners, respectively, $5.5 \mathrm{mmol} / \mathrm{L}$ and $6.2 \mathrm{mmol} / \mathrm{L}$ in male 24MTBers and male 100RUNners, respectively. On the contrary, the lowest pre-race level was $4.4 \mathrm{mmol} / \mathrm{L}$ in male SMTBers, $4.7 \mathrm{mmol} / \mathrm{L}$ in female SMTBers, $5.2 \mathrm{mmol} / \mathrm{L}$ in female 100RUNners, and we simultaneously found a significant increase in post-race plasma $\left[\mathrm{K}^{+}\right]$. Overall, despite the genders and the various numbers of racers in each ultra-disciplines $57.9 \%$ 24RUNners, $42.0 \%$ of 24MTBers, $16.7 \%$ 100RUNners and $6.3 \%$ of SMTBers showed pre-race hyperkalemia and 47.4 \% 100RUNners, $28.1 \%$ SMTBers, $20 \%$ 24MTBers and $16.7 \%$ 24RUNners developed post-race hyperkalemia.

A further interesting finding was that we found an association between pre-race levels of plasma $\left[\mathrm{K}^{+}\right]$and post-race $\mathrm{CK}$ levels in the present finishers. The ultraathletes with higher pre-race plasma $\left[\mathrm{K}^{+}\right]$developed post-race higher CK levels. It also appears that races with a higher occurrence of pre-race hyperkalemia tended to a lower occurrence of hyperkalemia post-race and conversely. However, there was no significant relationship between pre-race plasma $\left[\mathrm{K}^{+}\right]$and post-race plasma $\left[\mathrm{K}^{+}\right]$considering all present ultra-athletes. The presence of mild pre-race or post-race hyperkalemia in some present hyponatremic and normonatremic cases supports either excessive $\left[\mathrm{K}^{+}\right]$ingestion pre-race or during the race, ingestion of NSAID, reduced renal excretion or tissue breakdown as in rhabdomyolysis [42]. Notwithstanding, we found no association between postrace plasma $\left[\mathrm{K}^{+}\right]$and $C K$ concentration. We have to take into account that some results could be also caused by pseudohyperkalemia from leakage of $\left[\mathrm{K}^{+}\right]$from the intracellular space during or after blood sampling in field conditions [42].

\section{Plasma and urine creatinine concentrations}

Muscle injury releases creatine and increases blood creatinine as one of parameters of myocellular damage [31, 39]. Biochemical criteria for acute renal injury mean a blood creatinine concentration more than $2.0 \mathrm{mg} / \mathrm{dL}$ and 1.5 times of the estimated baseline [27, $31,39]$. Post-race plasma and urine creatinine significantly increased and creatine clearance decreased in all 
cases with rhabdomyolysis (cases 1-6), in the hyponatremic and the normonatremic group and in both genders in all present different races. Current cases 1 and 2 with $\mathrm{EAH}$ and rhabdomyolysis developed an average post-race creatinine level of $0.95 \mathrm{mg} / \mathrm{dL}(1.0 \mathrm{mg} / \mathrm{dL}$ and $0.8 \mathrm{mg} / \mathrm{dL})$. In accordance to the study of Bruso et al. [6], their average level of post-race blood creatinine in cases with EAH and rhabdomyolysis was higher than in the present study. Three cases in the study of Bruso et al. [6] developed acute renal failure with higher blood creatinine $(2.8 \mathrm{mg} / \mathrm{dL}$ to $4.9 \mathrm{mg} / \mathrm{dL}$ ) than two cases without renal failure $(1.1 \mathrm{mg} / \mathrm{dL}$ to $1.2 \mathrm{mg} / \mathrm{dL})$, however, without a difference in CK concentrations. Cases 3-6 with just rhabdomyolysis showed an average post-race creatinine level in the range from $1.0 \mathrm{mg} / \mathrm{dL}$ to $1.2 \mathrm{mg} / \mathrm{dL}$. In the study of Hoffman et al. [38], the range for blood creatinine levels was similar as in the present cases with rhabdomyolysis in the range from $1.1 \mathrm{mg} / \mathrm{dL}$ to $1.4 \mathrm{mg} / \mathrm{dL}$. The present hyponatremic group developed post-race creatinine concentration in the range from $0.8 \mathrm{mg} / \mathrm{dL}$ to $2.6 \mathrm{mg} / \mathrm{dL}$ and the normonatremic group from $0.7 \mathrm{mg} / \mathrm{dL}$ to $3.4 \mathrm{mg} / \mathrm{dL}$; however, without a significant difference between both groups.

Present creatinine levels above the upper limit of normal were only found in one hyponatremic male SMTBer $(2.6 \mathrm{mg} / \mathrm{dL}$, plus $271.4 \%$ ) and one male normonatremic SMTBer $(3.4 \mathrm{mg} / \mathrm{dL}$, plus $240.0 \%$ ) from a total of 113 ultra-athletes. The 42-year old hyponatremic male SMTBer (post-race plasma $\left[\mathrm{Na}^{+}\right] 134 \mathrm{mmol} / \mathrm{L}$ ) developed also high pre- and post-race levels of plasma $\left[\mathrm{K}^{+}\right](5.5$ and $5.7 \mathrm{mmol} / \mathrm{L})$, minus 3.8 percentage change in body mass; the highest percentage decrease (minus $74.1 \%$ ) of creatine clearance within all finishers; however with post-race CK just 1,197 U/L. The 26-year old normonatremic SMTBer showed plasma $\left[\mathrm{Na}^{+}\right] 140 \mathrm{mmol} / \mathrm{L}$, preand post-race levels of plasma $\left[\mathrm{K}^{+}\right] 3.6$ and $4.9 \mathrm{mmol} / \mathrm{L}$, plus $0.6 \%$ percentage change in body mass, the second highest percentage decrease (minus $70.4 \%$ ) of creatine clearance within all finishers; however similarly with post-race CK only 1,168 U/L. Both current cases presented post-race without the development of renal failure and the necessity of a medical treatment. Neumayr et al. investigated the effect of marathon cycling on renal function in recreational and professional road cyclists [22, 23] with no evidence for a significant skeletal muscle damage and a reduced renal perfusion responsible for the slight impairment of renal function after marathon cycling [22]. In a study of the 38 recreational male marathon cyclists the increases in plasma creatinine were $20 \%$, the decrease of creatinine clearance was similar $18 \%$ [22]. In 16 professional road cyclists $(525-\mathrm{km}$ race), plasma creatinine rose by $33 \%$, the decrease of creatine clearance was $25 \%$ [23]. Two multi-stage mountain bikers in the present study developed the highest levels of post-race plasma $\left[\mathrm{K}^{+}\right]$and the lowest concentrations of creatine clearance; however, both with low post-race CK concentrations. Moreover, these hyponatremic multi-stage bikers showed a higher percentage increase in post-race CK levels than normonatremic biker. In the study of Hoffman et al. [41], $4 \%$ of their ultra-runners met the criteria for injury (i.e. blood creatinine 2.0 times of the estimated baseline) and $29 \%$ for risk (i.e. blood creatinine 1.5 times of the estimated baseline) of acute renal injury and those meeting the injury criteria had higher CK concentrations. Nevertheless, very few runners seek or require medical treatment for acute renal injury [41]. In all present SMTBers plasma creatinine rose by $33.3 \% \pm$ 64.8 in men and by $21.9 \pm 15.7 \%$ in women with minus $17.4 \pm 19.3 \%$ change in male creatine clearance and minus $19.8 \pm 10.8 \%$ change in female creatine clearance and their post-race creatine levels and post-race percentage changes in $\mathrm{CK}$ were the lowest from all different ultra-disciplines in the present study. These data confirm that the strains of ultramarathon cycling regardless these two presented cases did not influence their renal function. Even though, Khalil et al. [43] suggested that acute renal failure is defined with serum creatinine $>3$-fold from baseline, or $>4 \mathrm{mg} / \mathrm{dL}$ with an acute rise of $0.5 \mathrm{mg} / \mathrm{d} \mathrm{L}$ or greater. The present multi-stage mountain bikers fulfilled these conditions; they did not seek or required medical treatment for acute renal injury. In the present study, correlations between post-race CK values and plasma creatinine, urine creatinine or creatine clearance values were not found.

\section{Limitations}

We have to take into account that the number of present ultra-athletes in various disciplines and gender representation was different. Further we did not observe a urine dipstick for a urine colour, blood and myoglobin. However, direct measurement of myoglobin is less reliable and not clinically useful [44]. Moreover, increased CK levels are associated with increased blood myoglobin [7, 38]. Hereafter, a development of rhabdomyolysis can delay several hours after the occurring of EAH due to the changes in intracellular $\left[\mathrm{K}^{+}\right]$and the restoring of cellular volume [14]. Peak CK level is about 48 to $96 \mathrm{~h}$ after its presentation [44]. In the marathon cyclists renal parameters remained elevated during $24 \mathrm{~h}$ of recovery $[22,23]$. We had no possibility to observe ultra-athletes one or more days after the race; otherwise the danger of rhabdomyolysis could be higher.

\section{Practical applications}

The value of $\mathrm{CK}$ as a prognostic tool is limited, due to the wide inter-individual range of CK levels. In situations where the diagnosis of EAH is uncertain, fluid restriction 
during or after the race is contraindicated in case of dehydration and rhabdomyolysis with impending acute kidney injury $[8,27,29,41]$.

\section{Conclusions}

The present hyponatremic ultra-athletes tended to develop exercise-induced rhabdomyolysis more than normonatremic ultra-athletes. Ultra-runners tended to develop rhabdomyolysis more than mountain bikers. The present work does not clarify the mechanism that might be involved in the suggested link between rhabdomyolysis and EAH.

\section{Consent}

Written informed consent was obtained from all testing subjects for the publication of this report and any accompanying images.

\section{Competing interests}

The authors declare that they have no competing interests.

\section{Authors' contributions}

DCH, BK and TR developed the objectives of the study and intervention, DCH managed recruitment and data collection, TR supported a laboratory processing of samples, DCH and AZ participated in the practical measurement in all field studies, DCH and IT performed statistical analysis; DCH, BK, IT, JN and TU lead the drafting of the manuscript, interpreted the findings and critically reviewed the manuscript. All authors read and approved the final manuscript.

\section{Acknowledgements}

The authors gratefully acknowledge the athletes for their splendid cooperation without which this study could not have been done. We thank the organizers and the medical crew of the "Czech Championship 24-h mountain bike race" in Jihlava, the "Bike Race Marathon Rohozec 24 h" in Liberec, the "Sri Chinmoy Self-Transcendence Marathon 24-h race" in Kladno, the "Trilogy Mountain Bike Stage Race" in Teplice nad Metují and the "100-km running race" in Plzen for their generous support. A special thank goes to the laboratory staff of the University Hospital 'U Svaté Anny' in Brno, Czech Republic, for their efforts in analyzing haematological and biochemical samples even during the night-times. We thank Matthias Knechtle for his help in English translation.

\section{Author details}

${ }^{1}$ Centre of Sports Activities, Brno University of Technology, Brno, Czech Republic. ${ }^{2}$ Institute of Primary Care, University of Zurich, Zurich, Switzerland. ${ }^{3}$ Faculty of Forestry and Wood Sciences, Czech University of Life Sciences, Prague, Czech Republic. ${ }^{4}$ Faculty of Sports Studies, Masaryk University, Brno, Czech Republic. ${ }^{5}$ Institute of Experimental Biology, Faculty of Science, Masaryk University, Brno, Czech Republic. ${ }^{6}$ Center for Adult Medicine in Bohunice, University Hospital Brno, Brno, Czech Republic.

Received: 9 June 2015 Accepted: 17 June 2015

Published online: 25 June 2015

\section{References}

1. Skenderi KP, Kavouras SA, Anastasiou CA, Yiannakouris N, Matalas AL. Exertional rahabdomyolysis duri ng a 246-km continuous race. Med Sci Sports Exerc. 2006;38(6):1054-7.

2. Clarkson PM. Exertional rhabdomyolysis and acute renal failure in marathon runners. Sports Med. 2007;37(4-5):361-3.

3. Lang G. Medical findings in the Western States 100 mile. Ultrarunning. 1984;3:14-8.

4. Matin P, Lang G, Carretta R, Simon G. Scintigraphic evaluation of muscle damage following extreme exercise: concise communication. J Nucl Med. 1983;24:308-11.

5. Lind RH, Eisenbund E, Lang G, Ashcraft M. CPK studies in Western States 100 mile endurance runners. Ultrarunning. 1996;48-50.
6. Bruso JR, Hoffman MD, Rogers IR, Lee L, Towle G, Hew-Butler T. Rhabdomyolysis and hyponatremia: A cluster of five cases at the 161-km 2009 Western States Endurance Run. Wilderness Environ Med. 2010;21(4):303-8.

7. Hoffman MD, Ingwerson JL, Rogers IR, Hew-Butler T, Stuempfle KJ. Increasing creatine kinase concentrations at the $161-\mathrm{km}$ Western states endurance run. Wilderness Environ Med. 2012;23:56-60.

8. Hoffman MD, Stuempfle KJ, Sullivan K, Weiss RH. Exercise-associated hyponatremia with exertional rhabdomyolysis: importance of proper treatment. Clin Nephrol. 2015;83:235-42.

9. Noakes T. Waterlogged. Human Kinetics: The serious problem of overhydration in endurance sports. New Zealand; 2012.

10. Kupchak BR, Kraemer WJ, Hoffman MD, Phinney SD, Volek JS. The impact of an ultramarathon on hormonal and biochemical parameters in men. Wilderness Environ Med. 2014;25(3):278-88.

11. Seedat YK, Aboo N, Naicker S, Parsoo I. Acute renal failure in the "Comrades marathon" runners. Ren Fail. 1989;11:209-12.

12. Kim HJ, Lee YH, Kim CK. Biomarkers of muscle and cartilage damage and inflammation during a 200 km run. Eur J Appl Physiol. 2007;99:443-7.

13. Fellmann $N$, Sagnol M, Bedu M, et al. Enzymatic and hormonal responses following a $24 \mathrm{~h}$ endurance run and a $10 \mathrm{~h}$ triathlon race. Eur J Appl Physiol. 1988:57:545-53.

14. Ellis C, Cuthill J, Hew-Butler T, George SM, Rosner MH. Exercise-associated hyponatremia with rhabdomyolysis during endurance exercise. Phys Sports Med. 2009;37(1):126-32.

15. Hoffman MD,Stuempfle KJ. Sodium supplementation and exercise-associated hyponatremia during prolonged exercise. Med Sci in Sports \& Exerc. 2014: [Epub ahead of print]

16. Hoffman MD, Fogard K, Winger J, Hew-Butler T, Stuempfle KJ. Characteristics of those with exercise-associated hyponatremia after a 161-km run. Res Sports Med. 2013;21:164-75.

17. Boulter J, Noakes TD, Hew-Butler T. Acute renal failure in four Comrades marathon runners ingesting the same electrolyte supplement: coincidence or causation? S Afr Med J. 2011;101(12):876-8.

18. Irving RA, Noakes TD, Raine RI, van Zyl SR. Transient oliguria with renal tubular dysfunction after a $90 \mathrm{~km}$ running race. Med Sci Sports Exerc. 1990;22:756-61.

19. Zyl-Smit R, Mills P, Vogelpoel L. Unrecognized acute renal failure following the Comrades marathon. S Afr Med J. 2000;90:39-40.

20. MacSearraigh ET, Kallmeyer JC, Schiff HB. Acute renal failure in marathon runners. Nephron. 1979;24:236-40.

21. Schiff HB, MacSearraigh ET, Kallmeyer JC. Myoglobinuria, rhabdomyolysis and marathon running. QJM. 1978;47:463-72.

22. Neumayr G, Pfister R, Hoertnagl H, Mitterbauer G, Getzner W, Ulmer H, et al. The effect of marathon cycling on renal function. Int J Sports Med. 2003;24(2):131-7.

23. Neumayr G, Pfister R, Hoertnagl H, Mitterbauer G, Prokop W, Joannidis M. Renal function and plasma volume following ultramarathon cycling. Int J Sports Med. 2005;26(1/02):2-8.

24. Pearce PZ. Statin-induced myopathy in a competitive amateur cyclist. Curr Sports Med Rep. 2008;7(3):149-51.

25. Shumate JB, Brooke MH, Carroll JE, Davis JE. Increased serum creatine kinase after exercise: a sex-linked phenomenon. Neurology. 1979;29:902-4.

26. Bosch X, Poch E, Grau JM. Rhabdomyolysis and acute kidney injury. N Engl J Med. 2009;361:62-72.

27. Hew-Butler T. Hyponatremia, rhabdomyolysis and renal failure. Is there a link? Marathon \& Beyond. 2013;17:70-80

28. Bontempo LJ. Rhabdomyolysis. In: Marx J, editor. Rosen's emergency Medicine: Concepts and clinical practise. 6th ed. Philadelphia, PA: Elsevier; 2006. Chapter 125.

29. Patel DR, Gyamfi R, Torres A. Exertional rhabdomyolysis and acute kidney injury. Phys Sportsmed. 2009;37:71-9.

30. Meijer AR, Fikkers BG, Keijzer MH, Engelen BGM, Drenth JPH. Serum creatine kinase as predictor of clinical course in rhabdomyolysis: a 5-year intensive care survey. Intensive Care Med. 2003;29:1121-5.

31. Sinert R, Kohl L, Rainone T, Scalea T. Exercise-induced rhabdomyolysis. Ann Emerg Med. 1994;23:1301-6.

32. Siegel AJ, Verbalis JG, Clement S, Mendelson JH, Mello NK, Adner M, et al. Hyponatremia in marathon runners due to inappropriate arginine vasopressin secretion. Am J Med. 2007;120:11-7.

33. Siegel AJ. Exercise associated hyponatremia: role of cytokines. Am J Med. 2006;119(7 Suppl 1):S74-78 
34. Swart RM, Hoorn EJ, Betjes MG, Zietse R. Hyponatremia and inflammation: the emerging role of interleukin-6 in osmoregulation. Nephron Physiol. 2011;118:45-51.

35. Putterman C, Levy I, Rubinger D. Transient exercise-induced water intoxication and rhabdomyolysis. Am J Kidney Dis. 1993;21(2):206-9.

36. Hoffman MD, Hew-Butler T, Stuempfle KJ. Exercise-associated hyponatremia and hydration status in 161-km ultramarathoners. Med Sci Sports Exerc. 2013;45(4):784-91.

37. Ward MM. Factors predictive of acute renal failure in rhabdomyolysis. Arch Intern Med. 1988;148:1553-7.

38. Clarkson PM, Eichner ER. Exertional rhabdomyolysis: does elevated blood creatine kinase foretell renal failure? Curr Sports Med Rep. 2006;5(2):57-60.

39. Clarkson PM, Kearns AK, Rouzier P, Rubin R, Thompson PD. Serum creatine kinase levels and renal function measures in exertional muscle damage. Med Sci Sports Exerc. 2006;38(4):623-7.

40. Thompson PD, Clarkson P, Karas RH. Statin associated myopathy. JAMA. 2003:289:1681-90.

41. Hoffman MD, Stuempfle KJ, Fogard K, Hew-Butler T, Winger J, Weiss RH. Urine dipstick analysis for identification of runners susceptible to acute kidney injury following an ultramarathon. J Sports Sci. 2013;31:20-31.

42. Lehnhardt A, Kemper MJ. Pathogenesis, diagnosis and management of hyperkalemia. Pediatr Nephrol. 2011;26:377-84.

43. Khalil P, Murty P, Palevsky PM. The patient with acute kidney injury. Prim Care. 2008;35(2):239-64.

44. O'Connor FG, Deuster PA. Rhabdomyolysis. In: Goldman L, Ausiello D, Arend W, et al., editors. Cecil Medicine. 23rd ed. Philadelphia. PA: Elsevier; 2007. p. $798-802$.

\section{Submit your next manuscript to BioMed Central and take full advantage of:}

- Convenient online submission

- Thorough peer review

- No space constraints or color figure charges

- Immediate publication on acceptance

- Inclusion in PubMed, CAS, Scopus and Google Scholar

- Research which is freely available for redistribution 